\title{
L. FEUERBACH Y LA TRADICIÓN FILOSÓFICA
}

\author{
Arsenio GINZO FERNÁNDEZ \\ Universidad de Alcalá
}

RESUMEN. El artículo muestra que la conciencia de la historicidad de la filosoffa ocupa un lugar central en la obra feuerbachiana. Desde este horizonte Feuerbach toma partido ante la historiografía filosófica, desde Kant hasta Hegel, y durante un tiempo se va a dedicar a escribir sobre la historia de la filosofía moderna. Feuerbach aborda la historia de la filosofía sin abandonar su condición de filósofo. Por ello cuando se convierta en crítico de Hegel, también va a cuestionar la historiografía filosófica hegeliana, por mucho que siga dependiendo de ella.

Palabras ClaVE. Feuerbach, Hegel, Historia de la filosofía, historicidad, filosoffa contemporánea.

ABSTRACT. This article shows that the problem of philosophy's historicity is a main point in Feuerbach's work. Feuerbach reflects indeed on the philosophical historiography from Kant to Hegel, and writes himself a history of the Modern Philosophy. Feuerbach faces up to history of philosophy without leaving his philosopher's condition. In this sense when Feuerbach becames a critical of the hegelian philosophy, he also will criticize the hegelian philosophical historiography.

KEYwORDS. Feuerbach, Hegel, History of Philosophy, historicity, contemporary Philosophy. 


\section{El problema de la historicidad en la filosofía feuerbachiana}

Al cumplirse el segundo centenario del nacimiento de L. Feuerbach (18041872), quisiéramos hacer algunas consideraciones sobre sus aportaciones historiográficas, que constituyen un tema importante en la primera fase de su obra, y asimismo, de una forma general, sobre su concepción de la historicidad de la filosofía que de alguna forma caracteriza al conjunto de la obra feuerbachiana. Sin duda algunos intérpretes ya han llamado la atención sobre este aspecto del pensamiento feuerbachiano, tratando de rectificar con ello la escasa relevancia que se le había prestado tradicionalmente ${ }^{1}$. Por nuestra parte quisiéramos sumarnos a esos esfuerzos, tratando de esclarecer ulteriormente algunos de los aspectos de dicha problemática.

Gadamer ha podido escribir con razón que cabe considerar como una característica fundamental de la filosofía del siglo XIX el que no resulte pensable al margen de la "conciencia histórica»" Tal va a ser en efecto la condición de la filosofía desde Hegel y Schleiermacher hasta Niezzsche y Dilthey, pasando desde luego por Feuerbach. De ahí no sólo el hecho de la proliferación de Historias de la filosofía a lo largo de este periodo sino además la circunstancia de que los filósofos propiamente tales vincularan explícitamente su reflexión filosófica a su inserción en el proceso de la historia de la filosofía.

En este punto sería preciso referirse a todo el proceso desencadenado por la filosofía crítica, por los románticos, especialmente por figuras señeras como Schelling y Schleiermacher, pero de una forma especial el gran referente, como es sabido, va a ser Hegel. Por ello G. Santinello y G. Piaia en su documentada Historia de las historias generales de la filosofia pueden con razón subtitular el volumen dedicado a este periodo: La edad hegeliana $a^{3}$. A pesar de lo problemáticos que puedan resultar muchos de sus planteamientos, no cabe duda que Hegel se nos presenta de una forma especial como el filósofo que indaga la presencia de la razón en la historia, tanto a nivel de la Historia universal como en lo que se refiere a

1 Véanse a este respecto M. W. Warstofsky, Ludwig Feuerbach, CUP, Cambridge 1977; A. Philonenko, La jeunesse de Feuerbach 1828-1841, Vrin, París 1990, 2 vol.; W. Jaeschke y F. Tomasoni (eds), Ludwig Feuerbach und die Geschichte der Philosophie, Akademie, Berlin 1998.

${ }^{2}$ H.- G. Gadamer, Fünf hermeneutische Studien, J. C. B. Mohr, Tübingen 1971, 86.

${ }^{3}$ G. Santinello y G. Piaia (eds), Storia delle storie generali della filosofia 4. L'età hegeliana, Antenore, Padova 1995. 
aquella dimensión más íntima, la historia de la filosofía. Por ello si la filosofía hegeliana ha influido profundamente en el destino de la filosofía contemporánea, ello es cierto de una forma especial por lo que atañe a la historicidad de la filosofía. A este respecto incluso un pensador como Nietzsche se ve precisado a rendir homenaje a su manera a Hegel: «Nosotros los alemanes somos hegelianos, aun cuando no hubiera existido Hegel ${ }^{4}$.

Una manifestación particular del influjo de Hegel aparece en la proliferación de Historias de la filosofía escritas por una serie de autores salidos de su escuela, y que en la estela de lo realizado magistralmente por el maestro, muestran su voluntad de enfrentarse también ellos al sentido del pasado filosófico, incluido el más reciente, condicionado por la irrupción epocal de Hegel y su escuela. Sus nombres son bien conocidos para los estudiosos de esta época: C. L. Michelet que aparte de ser el autor de una importante Historia de la filosofía fue el editor de las lecciones hegelianas sobre dicho tema. J. E.Erdmann que además de escribir una Historia de la filosofía moderna ofrece un relato muy detallado acerca de la historia de la filosofía a partir de la muerte de Hegel. Cabe recordar que Feuerbach va a escribir una recensión de la Historia de la filosofía moderna de Erdmann y va a manifestar tanto su acuerdo como sus discrepancias con la misma, especialmente por lo que atañe al comienzo de la filosofía moderna. Además K. Rosenkranz, el autor de la famosa biografía sobre Hegel, nos deja también importantes estudios sobre historia de la filosofía. Por último habría que señalar asimismo a autores como K. Fischer y a E. Zeller.

Dentro de este horizonte, escuetamente evocado, Feuerbach se va a mostrar tempranamente sensible no sólo de una forma general por lo que atañe a la historicidad de la filosofía como tal sino que en el proceso de su maduración filosófica se va a ocupar ampliamente de escritos de carácter historiográfico. En 1833, el año en que aparece póstumamente la Historia de la filosofía de Hegel, Feuerbach va a publicar también su Geschichte der neueren Philosophie von Bacon von Verulam bis Benedikt Spinoza, como primera aportación a su proyecto de una historia de la filosofía moderna. A ella va a seguir en 1837 un estudio monográfico sobre Leibniz Geschichte der neueren Philosophie. Darstellung, Entwicklung und Kritik der Leibnizischen Philosophie. Al año siguiente aparecerá un

${ }^{4}$ KSA III, 599. 
nuevo estudio monográfico, en este caso sobre P. Bayle, con el título: Pierre Bayle nach seinen für die Geschichte der Philosophie und Menschheit interessantesten Momenten dargestellt und gewürdigt. A estas tres aportaciones a la historia de la filosofía moderna, publicadas por el propio Feuerbach, habría que añadir el volumen publicado póstumamente por E. Thies en 1974 con el título Vorlesungen über die Geschichte der neueren Philosophie (1835/36) que tiene como base las lecciones sobre historia de la filosofía moderna impartidas por Feuerbach en la Universidad de Erlangen a mediados de los años 30. Finalmente habría que referirse a las frecuentes tomas de posición que el autor lleva a cabo a lo largo de su obra sobre este tema, especialmente por lo que a Hegel se refiere, $y$ asimismo al material inédito que pueda ir publicándose en el marco de las Gesammelte Werke.

Por innegables que sean las limitaciones, sobre todo a partir de un determinado momento de su evolución intelectual, que el naturalismo feuerbachiano supone para su valoración de la historia, no por ello deja de ser cierto que Feuerbach tuvo una clara conciencia de la historicidad de la filosofía, y en definitiva de la existencia humana, de forma que la filosofía feuerbachiana no resultaría comprensible si margináramos este aspecto de su obra. Sin duda en el horizonte de la filosofía posthegeliana, Feuerbach va a asumir abiertamente su condición de heraldo, de portavoz de una filosofía del futuro, de una "nueva" filosofía, pero ello no se produce al margen de la dinámica de la filosofía anterior que estaría exigiendo precisamente ese nuevo rumbo en la marcha de la filosofía. Quisiéramos contribuir un poco, en las páginas siguientes, a una mejor comprensión de algunos aspectos fundamentales de la visión historiográfica de Feuerbach, y en general de la centralidad de la historicidad de la filosofía en su obra.

\section{La relevancia de la historicidad de la filosofía en la obra feuerbachiana}

Hegel había subrayado el hecho de que la última filosofía, la filosofía del presente, habría de ser concebida como el resultado de todas las anteriores, de forma que la filosofía actual se presentaría como la "herencia" y el "resultado" del trabajo de todas las generaciones precedentes ${ }^{5}$, pues lo que somos no lo somos

${ }^{5}$ G. W. F. Hegel, Einleitung in die Geschichte der Philosophie, F. Meiner, Hamburg 1966, 21. 
al margen de la historia. He aquí una convicción que penetra también la obra feuerbachiana a pesar de todo el distanciamiento crítico frente a Hegel, que poco a poco se va a ir haciendo patente en la misma. Echando una mirada retrospectiva, a la altura de 1848 , sobre el sentido de su obra, Feuerbach va a poder afirmar con razón: «Yo no pertenezco por cierto a aquéllos para quienes un Kant y un Fichte, un Lutero y un Lessing, un Goethe y un Hegel habrian vivido y actuado en vano" ". Por supuesto esta lista podría ser fácilmente ampliada, pero aquí lo que interesa ante todo es la conciencia feuerbachiana acerca de su profunda inserción en la historia.

Esta conciencia se expresa reiteradamente a lo largo de la obra feuerbachiana. He aquí alguna toma de posición a este respecto. Cuando el joven Feuerbach, una vez presentada su tesis doctoral, tiene la oportunidad de impartir en la Universidad de Erlangen un ciclo de lecciones sobre Lógica y Metafísica es perfectamente consciente de utilizar el término Lógica en una acepción distinta de la habitual para situarse más bien en el horizonte de la acepción hegeliana que Feuerbach, con una serie de matizaciones, no duda en hacer suya, al menos por lo que atañe a su espíritu. El abordar la Lógica como Metafísica no es para Feuerbach fruto del capricho sino resultado de un proceso histórico, una situación que remite inevitablemente a la historia de la filosofía como horizonte desde el que resultaría comprensible. El exponer «la Lógica en su acepción como Metafísica constituye un resultado necesario de la presente historia de la filosofía. Por tanto la introducción más adecuada a la Lógica consiste en la exposición de la historia de la filosofiar" ${ }^{7}$.

Con ello Feuerbach se situaba tempranamente en un horizonte interpretativo en el que se iba a volver a situar en momentos decisivos de su obra. Así ocurre por ejemplo en la forma como concibe su obra más emblemática, Das Wesen des Christentums, con su tesis central de que la esencia de la Teología es la Antropología. Feuerbach por un lado reivindica su relevancia personal en la concepción y elaboración de la obra, pero por otro reconoce también que dicha obra no puede menos de concebirse como resultado de toda una dinámica histórica.

${ }^{6}$ L. Feuerbach, “Verhältnis zu Hegel», en Deutsche Zeitschrift für Philosophie 30(1982), 509 (ed. de W. Schuffenhauer).

7 L. Feuerbach, Gesammelte Werke (ed. de W. Schuffenhauer), X, 158-159. Salvo indicación en contrario citaremos conforme a esta edición. En adelante: G. W. 
A pesar de la autonomía que Feuerbach quiere atribuir a dicha obra, reconoce a continuación que ella ha de ser concebida a la vez como «una consecuencia necesaria de la historia»" ${ }^{8}$, señalando enfáticamente que el ensayo sobre la esencia del Cristianismo es "el resultado verdadero, hecho carne y sangre de la filosofia ante-

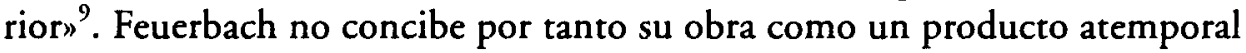
sino como plenamente inserta en la dinámica histórica. La publicación de esta obra feuerbachiana vendría a constituir un momento epocal de la historia: la confesión abierta de que la esencia de la Teología es la Antropología vendría a constituir para Feuerbach un momento especialmente cualificado del devenir histórico: «el necesario punto de viraje de la historia» ${ }^{10}$. La obra feuerbachiana subrayaría así su vinculación con la historia.

En realidad la propia dinámica de la experiencia religiosa conduciría asimismo a este desenlace. Lo mismo que su coetáneo A. Comte, el ilustrado posthegeliano que es Feuerbach no duda en integrar la religión en la historia espiritual de la humanidad. La religión vendría a ser el "primer" autoconocimiento del hombre, su autoconocimiento «indirecto", dado que el hombre comienza situando su ser "fuera de sí" antes de encontrarlo en sí mismo, de forma que su propio ser se le presenta inicialmente como un ser distinto. Pero también aquí el proceso histórico tendría la virtualidad de ir desenmascarando la autocomprensión inicial del hombre: «El proceso histórico de las religiones consiste por tanto en que lo que a las primeras religiones se les presentaba como algo objetivo sea reconocido como algo subjetivo, es decir, lo que fue considerado y adorado como Dios sea reconocido ahora como algo humano" ${ }^{11}$. Por ello Feuerbach no duda en afirmar que todo progreso en la conciencia religiosa constituye una forma más profunda de autoconocimiento, reafirmando así su concepción socrática de la dinámica de la historia.

También desde este punto de vista Feuerbach va a conceder una particular relevancia a la Modernidad. Este periodo desempeña un papel decisivo a través del despliegue y mutua complementación del doble principio moderno de la subjetividad: el religioso y el filosófico. Su desarrollo histórico habría puesto de mani-

\footnotetext{
G. W. V, 24.

9 G. W. V. 16.

${ }^{10}$ G. W. V, 444.

11 G. W. V, 47.
} 
fiesto el desenlace que Feuerbach andaba buscando: la mostración de que la esencia de la Teología es la Antropología. Con especial nitidez se muestra a este respecto en los Grundsätze der Philosophie der Zukunft: «El cometido de los tiempos modernos consistió en el desarrollo y la humanización de Dios - la transformación y disolución de la Teología en la Antropologian ${ }^{12}$. El protestantismo y la filosoffa especulativa, en su mutua complementación, habrían llevado a cabo este proceso. Mientras que el primero habría protagonizado esa disolución desde el punto de vista religioso y práctico, la segunda por el contrario habría llevado a cabo la disolución racional o teórica. Feuerbach concibe su obra en íntima conexión con ese complejo proceso histórico gestado a lo largo de la Modernidad.

Feuerbach se iba a convertir asi en un portavoz cualificado de aquella situación posthegeliana en la que la filosofia por una parte se siente heredera de un largo proceso histórico y por otra tiene la conciencia más o menos confusa de estar iniciando una nueva época, perfilando la filosofia del futuro, tal como vendría a decir Feuerbach ${ }^{13}$. Tempranamente se abre Feuerbach a esta doble mirada: la herencia del pasado y la apertura al futuro, considerando la necesidad de ir más allá de su época. Ante la escisión, propia de las épocas de transición, entre los partidarios del pasado y los del futuro, Feuerbach no vacila en situarse del lado de estos últimos, pero sin derivar por ello en una concepción ahistórica. Sea suficiente con recordar aquí el escrito juvenil Gedanken über Tod und Unsterblichkeit en los que una visión histórica de la problemática abordada se aúna con una apertura a un nuevo periodo en la historia espiritual de la humanidad, al uglorioso día del futuro" ${ }^{14}$. Varios años más tarde, al volver su mirada a este escrito juvenil, Feuerbach va a corroborar esa apertura al futuro como algo que lo habría caracterizado. Ese escrito habría dado expresión al "presentimiento» de una nueva época ${ }^{15}$.

Va a ser, no obstante, después de la publicación de Das Wesen des Christentums cuando Feuerbach va a redactar una serie de esbozos de una nueva filo-

12 G. W. IX, 265.

${ }^{13}$ Cf. W. Jaeschke (ed.), Philosophie und Literatur im Vormärz. Der Streit um die Romantik (1820-1854), F. Meiner, Hamburg 1995, 1 ss; L. Feuerbach, Entwïrfe zu einer Neuen Philosophie (ed. de W. Jaeschke y W. Schuffenhauer), F. Meiner, Hamburg 1996, VII ss.

${ }^{14}$ G. W. I, 198.

is Véase carta del 3.10.1869 a Math. F. Wendt. 
sofia ${ }^{16}$ en los que se intenta perfilar una filosofía del futuro. Pero Feuerbach va a seguir insistiendo ahora en que esa nueva filosofía no puede comprenderse al margen de su inserción en la historia. La nueva filosofía, escribe Feuerbach en los Grundsätze der Philosophie der Zukunft, surge a partir de la anterior, movida por una especie de "necesidad histórica»" ${ }^{17}$, de forma que cabría hablar de que la nueva filosofía surge de la antigua como una especie de "resultado necesario" ${ }^{18}$, produciéndose así toda una tensión dialéctica, de carácter epocal, entre la referencia a la tradición filosófica y la iniciación de un nuevo periodo histórico en la historia de la filosofía.

Pero Feuerbach no sólo estaba convencido de que esa tradición filosófica terminaba dando paso al nacimiento de una nueva filosofía, la filosofía del futuro sino de que en definitiva se estaba asistiendo al nacimiento de una nueva fase en la historia de la humanidad como tal. Feuerbach no dudaba de que en su tiempo se estaba dirimiendo algo más que un trasunto filosófico. Por el contrario mostraba su convicción de que se estaban abordando cuestiones que iban más allá del ámbito filosófico como tal e incidían de una forma directa en el corazón de la humanidad.

Por ello si el conjunto de la obra feuerbachiana aparece jalonada por una serie de tomas de conciencia acerca del carácter epocal de su momento histórico, también aparece acompañado por referencias constantes a la convicción de que la humanidad como tal se encontraba iniciando un nuevo periodo histórico. Sin duda Feuerbach siempre se esforzó por abrir el universo filosófico a las inquietudes y problemas de la humanidad como tal. Su primer esfuerzo, va a escribir, consistió en convertir la filosofía en asunto de la humanidad ${ }^{19}$. En efecto ya en la temprana carta a Hegel del 22.11.1828 se refiere Feuerbach a la situación filosófica del momento como algo que desbordaba los límites de la escuela para convertirse en trasunto de la humanidad como $\mathrm{tal}^{20}$. Esta apertura de la filosofía a las inquietudes de la humanidad se aúna con la convicción de que se estaba iniciando un nuevo periodo en la historia de la humanidad.

${ }^{16}$ Cf. L. Feuerbach, Entwürfe zu einer Neuen Philosophie.

17 G. W. IX, 314.

18 G. W. IX, 335.

${ }^{19}$ G. W. X, 180.

${ }^{20}$ G. W. XVII, 105. 
Basten aquí algunas referencias puntuales a esas tomas de conciencia. Ya en la mencionada carta a Hegel, Feuerbach se refería al imperativo de la época de dar paso a una "segunda historia», a una "segunda creación", siendo necesario transformar para ello el fundamento de la historia anterior, dando paso al surgimiento de un nuevo periodo de la Historia universal. Tal era el imperativo que se presentaba a los ojos de Feuerbach ante la consumación hegeliana de la historia de la filosofía, una filosofía hegeliana ya de por sí integradora de las grandes experiencias de la historia de la humanidad, tal como muestra la Fenomenología del espiritu.

No menos decidida va a ser la toma de posición feuerbachiana en aquel escrito juvenil que entre otras cosas iba a condicionar profundamente el destino de su autor. Nos estamos refiriendo obviamente al ensayo Gedanken über Tod und Unsterblichkeit de 1830 en el que se abordaba una temática cuya relevancia iba más allá de un debate académico, intrafilosófico, afectando por el contrario convicciones que incidían profundamente en la conciencia de la humanidad. A este respecto Feuerbach considera que esta última se encuentra en un punto de viraje en su historia espiritual. Por ello escribe enfáticamente: "A aquél que comprende el lenguaje en que habla el espíritu de la Historia universal, no se le puede ocultar que nuestra época actual constituye la clave de bóveda de un gran periodo en la historia de la humanidad, y el punto de partida de una nueva vida espiritual ${ }^{21}$. Feuerbach va a volver varias veces sobre este tema a lo largo de su obra, corroborando su temprana convicción de que se estaba iniciando una nueva época.

Si a través del hilo conductor del cuestionamiento de la inmortalidad individual el joven Feuerbach se abría a la idea de un nuevo periodo en la historia espiritual de la humanidad, no podía por menos de hacerlo también al abordar la problemática de Das Wesen des Christentums con su tesis central de que la esencia de la Teología es la Antropología. Según queda apuntado, la confesión abierta de esa tesis constituye para Feuerbach el necesario punto de viraje de la historia. De la Historia universal, subraya Feuerbach, pues también aquí estaríamos ante una cuestión que desbordaría el ámbito filosófico, para insertarse en el núcleo mismo de los problemas que inquietaban a la humanidad en cuanto tal.

${ }^{21}$ G. W. I, 196-197. 
Especialmente nítido y clarificador resulta un breve ensayo titulado Notwendigkeit einer Veränderung que pertenece a la serie de opúsculos publicados a continuación de Das Wesen des Christentums, en los que Feuerbach traza un esbozo de una nueva filosofía. En dicho ensayo Feuerbach se refiere abiertamente al carácter epocal de su momento histórico y distingue claramente entre una filosofía que coincide con las anteriores formando parte de una época común, una filosofía que debe su existencia sólo a una necesidad filosófica, tal como sería el caso de la filosoffa de Fichte frente a la de Kant, y otra filosofía por el contrario que surge de un nuevo periodo de la historia de la humanidad, y que por tanto no respondería meramente a una necesidad filosófica sino a una necesidad de la humanidad, conectando así de una forma inmediata con la historia de la humanidad $^{22}$. Por el contrario la primera forma de filosofía pertenecería directamente a la historia de la filosoffa y sólo de una forma indirecta a la historia de la humanidad. En este contexto Feuerbach se pregunta abiertamente: ¿se trata tan sólo de una nueva filosoffa o por el contrario nos encontramos ante un nuevo periodo de la historia de la humanidad? Feuerbach estaba convencido de que las importantes transformaciones que estaban teniendo lugar implicaban que habría que decidirse por la segunda alternativa. Con ello expresaba su profunda conciencia acerca de la historicidad de todas las formas de existencia que configuraban a su época. Sus trabajos sobre historia de la filosofía han de ser situados en este horizonte que se irá perfilando poco a poco en la evolución intelectual de Feuerbach. A pesar de todos sus límites el problema de la historicidad constituye un punto central del pensamiento feuerbachiano.

\section{Feuerbach y la historiografía filosófica de su tiempo}

Sin duda el tema especifico de la historiografia filosófica se encontraba en un momento especialmente relevante en aquel periodo, tal como queda ya apuntado, y Feuerbach es consciente de ello. Es de una forma especial la filosofía alemana, a partir de Kant, la que se señalaba especialmente en la tarea de buscar los criterios más adecuados para dialogar con la tradición filosófica. Un filósofo como Feuerbach, que no abdicará de su condición de filósofo al enfrentarse con la historia de la filosofía, sabe valorar el giro que la filosofía crítica supo imprimir a este respecto: «La filosofía crítica tiene el mérito de ser la primera en considerar

${ }^{22}$ L. Feuerbach, Entwürfe zu einer Neuen Philosophie, 119. 
la historia de la filosofía desde un punto de vista filosófico» ${ }^{23}$, y no como un repertorio de opiniones de todo tipo, que se convierte a menudo en una presentación de opiniones pintorescas y ridículas. Feuerbach no dudaba en poner como representante de esta última forma de presentar la historia de la filosofía a un autor como Ch. Meiners, que se situaba en el marco de la Populärphilosophie de la Ilustración tardía. A éste cabría añadirle otros representantes como Garve o Tidemann.

Frente a éstos reflejarían por el contrario una visión más satisfactoria kantianos como K. L. Reinhold cuyo estudio Über den Begriff der Geschichte der Philosophie utilizaba como criterio para abordar la historia de la filosofía el «sentido filosófico racional» ${ }^{24}$. El autor de las Briefe über die kantische Philosophie se esfuerza por extraer las virtualidades del kantismo para la historia de la filosofía en la medida en que lo considera como el "punto de vista más elevado de la historia", y que por tanto habría de ser tomado como referente para evaluar los demás sistemas filosóficos.

Otro representante de la historiografia de corte kantiano es J. Ch. Grohmann y su estudio Über den Begriff der Geschichte der Philosophie, que también parte de la convicción de que la filosofía crítica viene a constituir "la" filosofía de acuerdo con la que se habrían de evaluar los distintos sistemas filosóficos. Éstos no son deducidos de acuerdo con criterios externos sino siguiendo las leyes internas del conocimiento, como formas racionalmente necesarias del espíritu, en una relación histórica determinada por así decirlo a priori $^{25}$.

Feuerbach considera como un avance esta historiografia filosófica surgida en el horizonte de la filosofía crítica pero no le resulta satisfactoria sin más debido fundamentalmente al hecho de que una filosofía determinada es considerada como la "verdadera", como constituyendo la meta que de una u otra forma habrían aspirado a realizar los demás sistemas filosóficos. Así la doctrina kantiana acerca de los límites de la razón constituiría una especie de rasero ineludible al que habrían de someterse las demás filosofias. Ello explicaría a juicio de Feuerbach que incluso un autor como W. G. Tennemann en su monumental Geschichte der

\footnotetext{
${ }^{23}$ G. W. VIII, 44.

${ }^{24}$ Ibid.

25 Ibid.
} 
Philosophie, al que no duda en considerar como el más importante historiador de la filosofía de orientación kantiana, resulte "unilateral, uniforme y aburrida", pues en definitiva se recurriría, a la hora de valorar los modernos sistemas filosóficos, a las mismas explicaciones, fundamentaciones y objeciones ${ }^{26}$. En definitiva, la historiograffa filosófica de corte kantiano tendría a los ojos de Feuerbach el mérito de haber abordado la historia de la filosofía desde una óptica filosófica pero violentaría la consideración de los distintos sistemas filosóficos al tomar como referente una filosofia determinada que es considerada como «la» filosofia.

Desde esta perspectiva Feuerbach no puede menos de saludar la evolución filosófica que se produce en Alemania a continuación del kantismo. Ello iba a tener consecuencias importantes, por lo que se refería a la forma de concebir la historia de la filosofía. A este respecto Feuerbach se va a fijar en el significado de Schelling y de su escuela, y a su aportación a una nueva concepción de la filosofía y de su historia. La filosofía pierde ahora los límites e insuficiencias que la filosofía kantiana imponía a la consideración de los demás sistemas filosóficos, resultando de ello una consideración demasiado externa y violenta. Frente a tal punto de vista una obra como el Sistema del Idealismo transcendental de Schelling suponía para Feuerbach una apertura del horizonte filosófico, más allá de las limitaciones kantianas, que facilitaba asimismo una visión de la historia de la filosofía más libre. La idea de la filosofía se abre ahora a la idea de infinitud, y más en concreto a la idea de identidad absoluta de lo Ideal y lo Real, tal como aparece en el mencionado ensayo de Schelling.

Pero si Feuerbach celebra la superación de las limitaciones que para una concepción de la historia de la filosofía provenían de la filosofía kantiana, pronto va a observar que desde el horizonte schellinguiano van a surgir nuevos problemas. Desde la perspectiva de la identidad absoluta de lo Ideal y lo Real no se está en condiciones de señalar debidamente el carácter específico, particular de cada sistema filosófico. De esta forma la historia de la filosofía carecería de la debida precisión y diferenciación, quedando sus perfiles más bien diluidos en una especie de magma general. Tal sería el defecto fundamental de una obra como el Handbuch der Geschichte der Philosophie de Th. A. Rixner, una obra que desde otros puntos de vista es elogiada por Feuerbach.

${ }^{26}$ G. W. VIII, 45. 
Era preciso por tanto dar un paso más en la búsqueda de un enfoque satisfactorio de la historia de la filosofia, era preciso buscar la forma de dar precisión y concreción a la identidad absoluta de modo que fuera posible conciliar la universalidad de la idea con su manifestación particular en la realidad. Tal sería el paso dado por Hegel que va a causar un profundo impacto en Feuerbach. A pesar de todo el distanciamiento crítico respecto al maestro, que empezó a manifestarse tempranamente, la historiografía filosofica hegeliana va a ejercer un influjo considerable en el pensamiento feuerbachiano.

Nos referiremos más adelante a ese distanciamiento crítico que Feuerbach va a manifestar de una forma paulatina respecto a la historiografía filosófica de Hegel. Aquí nos contentamos con subrayar que Feuerbach mostró poseer una comprensión profunda de la concepción historiográfica hegeliana, tal como muestra en su recensión de la Historia de la filosofía de su antiguo maestro. Para empezar Feuerbach señala claramente la centralidad de la historia en la filosofía de Hegel: «El concepto de historia en general es un concepto que se identifica con la idea fundamental de su filosofía" ${ }^{27}$, tan central que, apunta de momento tímidamente Feuerbach, el elemento de la unidad y de la simultaneidad que resultan tan relevantes en filosofías como la de Spinoza, quizá se retraigan más de lo debido en la filosofía hegeliana. En todo caso Feuerbach no duda que la consideración hegeliana de la historia de la filosofía no pierde de vista ni la "unidad» de la Idea en los distintos sistemas ni tampoco la "diferencia" y la particularidad de los mismos, ciertamente de una forma más satisfactoria de lo que era el caso en la filosofía de la identidad.

Dentro de este marco Feuerbach subraya una serie de rasgos que por una parte reflejan bien la concepción hegeliana de la historia de la filosofía y por otra condicionan de una forma importante la concepción feuerbachiana, a pesar del distanciamiento crítico que poco a poco se va a ir produciendo. He aquí algunos de esos rasgos:

1) Feuerbach subraya la «intimidad" con que Hegel aborda el estudio de los filósofos del pasado. Hegel no los interpreta como a extraños sino como a sus antepasados, familiares suyos. Por ello cabría decir que Hegel está «cabe sín al estudiar a Parménides y Heráclito, a Platón y Aristóteles. 
2) En este sentido cabría decir que la historia de la filosofía se ocupa en definitiva del presente, de lo actual dado que las formas posteriores de la filosofía conservan e interioirizan los "principios" de las filosofías anteriores en la profundidad del presente. Por ello el estudio de la historia de la filosofía también vendría a constituir él mismo un estudio de la filosofía.

3) La historia de la filosofía no puede ser por tanto para Hegel el estudio de opiniones y pensamientos contingentes, de carácter subjetivo, algo así como de ocurrencias ingeniosas que se hubieran ido expresando a lo largo de la historia. Para Hegel la historia de la filosofía ha de ser concebida como el desvelamiento progresivo de la verdad que encuentra su expresión más adecuada en la idea de desarrollo. Los distintos sistemas filosóficos constituyen por tanto momentos necesarios y racionales en el despliegue del conocimiento de la verdad.

4) A pesar de la lógica interna que muestra a los ojos de Hegel el desarrollo de la historia de la filosofía, a través de los distintos sistemas, la historia de la filosofía se encuentra a la vez en una estrecha relación con la Historia universal. Un mismo espíritu subyace a las distintas manifestaciones históricas, la especificidad de la filosofía consiste en expresarlo conceptualmente.

5) De todo ello Feuerbach no duda en concluir el lugar excepcional que compete a la Historia de la filosofía de Hegel en el análisis de la tradición filosófica: "Su historia es por consiguiente, de una forma indiscutible, la primera que constituye y garantiza un conocimiento real de la historia de la filosofía; el auténtico sentido, el concepto de los diferentes sistemas" ${ }^{28}$.

He aquí algunos de los rasgos fundamentales de la visión hegeliana destacados por Feuerbach. Una época brillante para la consolidación de la historia de la filosofía encontraba su culminación en la ingente aportación hegeliana. En su propia confrontación con la tradición filosófica, Feuerbach no podrá menos de acusar el fuerte impacto de la historiografía hegeliana pero a la vez

${ }^{28}$ G. W. VIII, 47. 
va a mostrar un grado de autonomía intelectual que no ofrecieron otros discípulos de $\mathrm{Hegel}^{29}$.

\section{Filosofía e historia de la filosofía según Feuerbach}

Hegel como gran filósofo que se enfrenta a la interpretación de la historia de la filosofía rehuye considerar a ésta como un tema de erudición estéril y superflua. Se esfuerza por el contrario por resaltar la dimensión filosófica de esa historia. Feuerbach por su parte puede ser considerado como la personalidad filosófica más relevante entre los discípulos de Hegel que se han ocupado de la historia de la filosofia . Por ello no se acerca tampoco a la historia de la filosofía como mero erudito sino que dialoga con ella para comprender mejor el presente y preparar el advenimiento de una nueva filosofía. Sin duda es justo destacar el nivel de información y documentación que está en la base de los trabajos historiográficos de Feuerbach ${ }^{30}$. Obviamente se trata de un aspecto imprescindible en toda labor historiográfica seria y Feuerbach ha sabido realizar satisfactoriamente esta tarea. Pero Feuerbach siempre rehuyó quedarse en un nivel meramente "erudito".

Tal como le escribe en 1839 a Carl Riedel: «Los escritos eruditos, es decir históricos no constituyen mi profesión; por el contrario, contradicen a mi ser» ${ }^{31}$. En la misma misiva Feuerbach va a rechazar como una impresión superficial el que se consideren sus trabajos historiográficos como "compilaciones eruditas", pues al realizarlos habría procurado no sacrificar el pensador al historiador, el interés filosófico al histórico. Feuerbach va a considerar más bien que en sus trabajos históricos había expresado, en última instancia, bajo "nombres ajenos" sus "propios pensamientos" ${ }^{32}$. Esto, como queda apuntado, no quiere decir que Feuer-

${ }^{29}$ Es curioso observar cómo un siglo más tarde M. Heidegger va a seguir opinando que la Historia de la filosofia de Hegel no sólo sería la primera sino propiamente la única que alcanza propiamente la meta señalada por Feuerbach: "La Historia de la filosofía de Hegel es hasta el momento la única historia de la filosoffa filosófica y lo seguirá siendo hasta que la filosofía piense históricamente, de una forma esencial, en un sentido todavía más originario, desde su pregunta fundamental más propia» (Id., Nietzsche I, Neske, Pfullingen 1961, 450).

${ }^{30}$ Cf. A. Philonenko, op. cit., 172.

${ }^{31}$ G. W. IX, 6.

32 G. W. X, 185. 
bach se hubiera ahorrado el trabajo de informarse y documentarse rigurosamente acerca de la temática a tratar sino que la finalidad última perseguida iba encaminada a una mejor comprensión del presente. Así se explicaría, por ejemplo, el hecho llamativo de que dedicara toda una monografía a un precursor de la Ilustración no tanto como ejercicio de erudición sobre una figura del pasado sino más bien para reivindicar su vigencia en la Alemania de Feuerbach.

Aunque con muchas lagunas, debido al carácter limitado de su actividad historiográfica, Feuerbach ha sabido protagonizar un diálogo profundo con la Modernidad filosófica, haciendo ver cómo la dinámica que la preside conduce poco a poco a su propio horizonte, al horizonte posthegeliano. Feuerbach sabe establecer un diálogo profundo y complejo, procurando desvelar la dimensión oculta de todo ese proceso histórico ${ }^{33}$. Respecto a todo ello parece convincente el punto de vista de M. W. Wartofsky cuando resalta el carácter socrático de la dialéctica feuerbachiana, en el sentido de que "el análisis y la crítica de los conceptos y teorías vendría a ser un proceso de autodescubrimiento y autotransformación " ${ }^{34}$. Dos son ante todo los grandes hilos conductores en la confrontación feuerbachiana con esa Modernidad filosófica: los avatares del llamado principio moderno de la subjetividad, tanto en su vertiente filosófica como religiosa, y sin cuyo análisis no sería posible comprender el magnum opus feuerbachiano, Das Wesen des Christentums. El otro va a ser la corriente naturalista, la revalorización de la sensibilidad, la experiencia y de las ciencias de la naturaleza a lo largo de los tiempos modernos, otro referente fundamental para la comprensión de la filosofía feuerbachiana.

De esta forma al oficiar como historiador de la filosofía moderna, Feuerbach no abdica de su condición de filósofo, por más que se trate de un filósofo que se encuentra aún en proceso de maduración, a la búsqueda de su identidad intelectual. A este respecto las distintas publicaciones de Feuerbach sobre la historia de la filosofía constituyen un buen referente para evaluar la evolución intelectual del autor. Así el primer volumen de su Historia de la filosofía se nos presenta como el más convencional, sobre todo su Introducción en la que Feuerbach mues-

${ }^{33}$ Cf. H.- J. Braun y otros (eds), Ludwig Feuerbach und die Philosophie der Zukunft, Akademie, Berlin 1990, 17 ss.

${ }^{34}$ M. W. Wartofsky, op. cit., VII. 
tra un excesivo mimetismo hacia los planteamientos hegelianos. El propio Feuerbach va a reconocer años más tarde esta circunstancia cuando le confiese a su amigo Ch. Kapp que había llegado a concebir una Introducción distinta de la actual en la que expresaría sus puntos de vista personales, pero que finalmente no se habría atrevido a plasmarla sobre el papel ${ }^{35}$.

No obstante, no todo es convencional en esta primera publicación historiográfica. Feuerbach reitera aquí la peculiar fascinación que experimentó en su juventud por la figura de Jacob Böhme, lo que sin duda contribuye a dar a su Historia un carácter peculiar. Feuerbach reafirma su admiración por un autor que a pesar de su modesta apariencia externa ocultaria "tesoros" en su interior. Se trataría de un místico, pero un místico que el joven Feuerbach ve luchando por alcanzar la libertad y el conocimiento puro y claro (35). Más "ortodoxo" resultaba por el contrario el entusiasmo por Spinoza, que se va a convertir en uno de los referentes más firmes del pensamiento feuerbachiano.

No menos relevante es la presencia de una valoración más positiva por parte de Feuerbach del problema de la sensibilidad. Sin duda esa valoración se encuentra todavía muy matizada en el primer volumen de la historia pero resulta ya claramente perceptible en el tratamiento de autores de tendencia empirista, especialmente en el caso de Francis Bacon, algo que a partir de ahora irá en aumento. Años más tarde, cuando escriba el prólogo de sus Obras completas, se va a referir expresamente Feuerbach a esta primera valoración del empirismo en el primer volumen de su Historia: «Por mucho que polemizaras contra los padres del empirismo, Bacon, Hobbes, Gassendi, en lo referente a la teoría del origen de las ideas y en otros puntos, los has tratado, no obstante, con un amor especial, sobre todo a Bacon y ya has declarado el empirismo como un «asunto de la filosofía" ${ }^{36}$. Sin duda por muchos que sean los reparos que subsistan, ya se aprecia en Feuerbach una actitud hacia Bacon que difiere de la mantenida por Hegel. Nos encontramos ahora ante una valoración afín, más receptiva.

Por otra parte también se observa, a pesar del estilo bastante convencional adoptado todavía por Feuerbach, una actitud más decidida, menos matizada que

\footnotetext{
${ }^{35}$ G. W. XVIII, 38.

${ }^{36}$ G. W. II, 160-161.
} 
la de Hegel, respecto al problema de la emancipación de la filosofía respecto a la teología, temática que también desempeña un papel central en la obra historiográfica de Feuerbach, y que cada vez se va a manifestar de una forma más abierta en sus trabajos historiográficos.

Después de este primer volumen habría que referirse a las Vorlesungen über die Geschichte der neuern Philosophie de 1835 que, según hemos indicado, permanecieron inéditas hasta tiempos recientes. En esta obra se observa una aproximación menos convencional a la filosofía moderna. Se había producido una evolución filosófica en Feuerbach y tal circunstancia condiciona asimismo su lectura de la filosofía moderna. Cabría destacar a este respecto la creciente relevancia que va a sumir la corriente naturalista que recorre el pensamiento moderno, comenzando por el naturalismo renacentista. Esto va a conducir a Feuerbach entre otras cosas a replantearse el problema del comienzo de la filosofía moderna. Feuerbach desplaza ahora esos orígenes a Italia, en concreto a sus pensadores naturalistas del Renacimiento. Tal opción le va a conducir por lo demás a polemizar con otro importante historiador de la escuela de Hegel que seguía más fielmente el punto de vista del maestro acerca del papel desempeñado por Descartes como iniciador de la filosofía moderna, a saber, E. Erdmann ${ }^{37}$.

Así como la filosofía griega había comenzado con las concepciones naturalistas de los jónicos, también la filosofía moderna habría de tener su comienzo en la filosofía de la naturaleza del Renacimiento. Varios son los autores italianos hacia los que Feuerbach dirige su atención, pero en primer lugar lo hace hacia G. Bruno por el que va a experimentar una fascinación especial. Tal como le escribe a B. Löw el mismo año 1835, Feuerbach no duda en considerar a Bruno como a su "amigo más íntimo ", como a su espíritu "más afín" ${ }^{38}$. Bruno vendría a ser el primer gran autor renacentista que expresaría de una forma convincente el espíritu de la nueva época y que habría muerto, lo mismo que Sócrates, como víctima y representante de los nuevos tiempos. El problema de la unidad, de la coincidencia de los contrarios, es aquella dimensión que Feuerbach quiere destacar en primer lugar en el pensamiento de Bruno. Sería precisamente esta idea la que propiamente convierte a Bruno en el iniciador de la filosofía moderna.

\footnotetext{
${ }^{37}$ G. W. X, 187.

${ }^{38}$ G. W. XVII, 217.
} 
Descartes por el contrario representaría una fase posterior del desarrollo filosófico, la de la oposición, la de la diferencia, en definitiva la fase de la crítica, fase a la que sin duda también Feuerbach concede una importancia primordial, pero que no podría ser, no obstante, la inicial. Descartes vendría a ser para Feuerbach una especie de Anaxágoras del pensamiento moderno ${ }^{39}$.

Tal orientación naturalista y reivindicadora de la sensibilidad le distanciaba sin duda de Hegel y de sus discípulos más ortodoxos, pero había, no obstante, otras cuestiones en las que el peso del maestro seguía siendo considerable. Tal es ante todo el enfoque filosófico de la historia de la filosofía, temática que Feuerbach va a plantear ahora de una forma mucho más abierta y explícita de lo que era el caso en el primer volumen de su Historia. Feuerbach declara abiertamente que quiere leer la historia de la filosofía como filosofía, dado que se trata de una exposición que se eleva por encima de las opiniones y concepciones subjetivas para situarse más bien en el plano de la necesidad.

Esta necesidad de los sistemas filosóficos es doble, aunque se produzca inseparadamente: externa e interna. En primer lugar un sistema filosófico estaría provisto de una necesidad externa en cuanto aparece en un tiempo determinado y en ese sentido aparece condicionado por factores como el nivel cultural, la forma de vida imperante y también por los sistemas filosóficos ya existentes. Pero esta necesidad externa encuentra su apoyatura en otra necesidad, en este caso de carácter interno, en la medida en que los sistemas filosóficos son manifestaciones particulares de la Idea absoluta e infinita, manifestaciones de la idea de Verdad. Los sistemas filosóficos serían por consiguiente necesarios «en la medida en que aparecen como autofundados en la idea de Verdad y constituyen modos como la Verdad no sólo puede ser aprehendida sino dado que ella puede ser aprehendida de ese modo, también debe en algún momento ser aprehendida así» ${ }^{40}$. La historia de la filosofía alcanzaría así toda su radicalidad y toda su relevancia filosófica. La historia de la filosofía vendría a ser también ella misma filosofía en cuanto despliegue de las distintas formas bajo las que puede ser aprehendida la Verdad como tal.

${ }^{39}$ L. Feuerbach, Vorlesungen über die Geschichte der neueren Philosopbie (1835-36), (ed. de E. Thies), Wiss. Buchgesellschaft, Darmstadt 1974, 49-50.

I0 Ibid., 11-12. 
Desde este horizonte el concepto de "desarrollo" desempeña en la historiografia feuerbachiana un papel central. De una forma especial Feuerbach va a tematizar este punto en su monografia sobre Leibniz, que él concibió como el segundo volumen de su Historia de la filosofia, y que sin duda constituye una aportación más madura a la historiografía filosófica. Los intérpretes de Feuerbach suelen coincidir a la hora de resaltar la relevancia de la monografia feuerbachiana en la historia de la recepción de la filosofía de Leibniz ${ }^{41}$. La obra de Feuerbach ha conseguido un mayor grado de madurez y fluidez, perdiendo por el contrario mimetismo y convencionalismo.

Por lo que concierne al concepto de desarrollo, Feuerbach va a considerar a la capacidad de desarrollo como un rasgo peculiar de lo que entendemos por filosofía. Por ello señala que al abordar la historia de la filosoffa se le ha de conceder la relevancia que le compete. Una historia de la filosoffa no ha de limitarse a la "actividad formal de la exposición" sino que ha de intentar poner de manifiesto la presencia de un desarrollo inmanente de los sistemas filosoficos. El siguiente pasaje describe bien cómo Feuerbach entendía por entonces ese concepto de desarrollo en la historia de la filosoffa: «El desarrollo es el desciframiento del verdadero sentido de una filosofia, el desvelamiento de lo que hay de positivo en ella, la exposición de su Idea en el seno de la forma determinada, temporalmente condicionada y finita, de esta Idea. La posibilidad del desarrollo es la Idea ${ }^{42}$. La Idea con su riqueza inagotable de formas bajo las que puede ser aprehendida.

Feuerbach, por tanto, como historiador de la filosofia no se queda en un nivel de consideración meramente erudito. Pretende llegar a lo que hay de esencial en esa filosoffa aunque para ello haya que ir más allá de la apariencia inmediata buscando más bien lo que está oculto y no dicho. La historia de la filosofía ha de tener presentes ciertamente los condicionamientos externos en cuyo horizonte se desenvuelve pero sin olvidar que en definitiva el verdadero objeto de la historia de la filosofía son las «acciones inmanentes del espíritu" que se rigen por una dialéctica peculiar: la inteligencia permanece cabe sí en lo otro: "La inteligencia

${ }^{41}$ Así se expresa, por ejemplo, A. Philonenko: «Si se tiene en cuenta el nivel entonces alcanzado por la historia de la filosofía como disciplina filosófica y científica, se trata de una obra magistral que supera, en nuestra opinión, todo lo que hasta entonces se había escrito sobre Leibniz" (Id., op. cit. I, 172).

${ }^{42}$ G. W. III, 4. 
no se sitúa, al alienarse, en un elemento extraño; el pensamiento se enajena para ser pensado de nuevo, no, pongamos por caso, para ser visto o creído; él permanece constantemente en su patria, en su origen" ". Feuerbach está convencido de que este tipo de consideraciones son especialmente oportunas al abordar un pensador como Leibniz en el que tan relevante resulta el problema del desarrollo de sus virtualidades interpretativas, pues el gran pensamiento de Leibniz vendría a ser la inmanencia del espíritu a sí mismo como fuente de sus ideas esenciales ${ }^{44}$.

En un nivel de mayor concreción cabría decir que la monografía sobre Leibniz le va a brindar la oportunidad a Feuerbach de abordar de nuevo el problema de la relación entre lo racional y lo empírico que ya había iniciado en el primer volumen de su Historia. La posición de Feuerbach está lejos entonces de poder calificarse de empirista sin más. Pero se observa en todo caso una valoración cada vez más positiva del significado filosófico del empirismo. A pesar de aquella autonomía del espíritu de que con razón habla Leibniz, cabría referirse al ugran significado histórico" del empirismo por haber restablecido los derechos de los sentidos, en cuanto medios del conocimiento, convirtiéndose la esfera de lo empírico en algo imprescindible, esencial ${ }^{45}$. Tal como escribe M. W. Wartofsky, la crítica de Leibniz a los planteamientos empiristas de Locke le sirve a Feuerbach como estímulo para proseguir su búsqueda de un empirismo más satisfactorio ${ }^{46}$.

Por otra parte aborda ahora de una forma más decidida el problema de la emancipación de la filosofía respecto a la teología. No duda que en este problema se encontraría precisamente el punto más débil de la construcción leibniziana. Feuerbach admiraba sin duda a Leibniz. No dudaba en considerarle un «genio universal sin igual», como el ansia de saber personificada y como la principal referencia literaria de su época ${ }^{47}$. No obstante, Feuerbach lamenta que el gran pensador no hubiera tenido la dicha de vivir en un momento histórico en el que el espíritu estuviera reconciliado consigo mismo sino que le hubiera correspondido vivir en una época en la que imperaba el dualismo entre la fe y la razón. Tal situación también habría afectado a Leibniz impidiéndole elaborar una filosofía

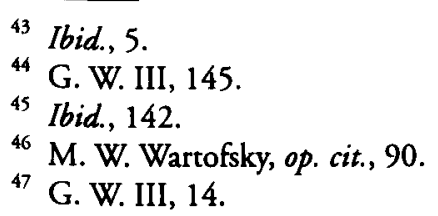


que fuera un todo homogéneo, al verse abocada a una serie de compromisos que le impiden llevar sus planteamientos filosóficos hasta el final ${ }^{48}$. De esta forma la búsqueda leibniziana de una armonización entre la fe y la razón se iba a convertir en piedra de escándalo para Feuerbach que se estaba distanciando de la Versöhnung hegeliana y que por el contrario se estaba acercando a los postulados radicales de la Ilustración.

Precisamente el tercer volumen de la Historia de la filosofía está consagrado a la figura de P. Bayle, lo que ciertamente distaba de ser una obviedad, pues se trataba de una figura "menor" de la historia de la filosofía moderna. Feuerbach es consciente de que su opción tiene algo de provocación y por ello comienza su monografía abordando esta circunstancia. Para nuestro propósito aquí lo más relevante es observar cómo la preocupación por el presente y el futuro condicionó el rumbo de los trabajos historiográficos de Feuerbach. Al ocuparse de Bayle Feuerbach quiere ocuparse de algo más que de un trabajo de erudición histórica. Pretende más bien criticar su presente histórico con ayuda de la tradición ilustrada. Por ello comienza reivindicando la vigencia de Bayle no sólo para la historia de la filosofía sino también, de forma significativa, para la historia de la humanidad como tal. Sin duda, tal como se ha señalado a menudo, Feuerbach veía en este momento en Bayle una especie de "alter ego".

Precisamente Feuerbach consideraba a Bayle como a uuno de los primeros y más excelentes luchadores en pro de la Ilustración, la humanidad y la tolerancia, libre de las ataduras de la fe tanto católica como protestante»" ${ }^{49}$. Sin duda Feuerbach deseaba conceder la palabra a Bayle después de haberse ocupado del autor de la Teodicea, pero la finalidad última era la crítica y la comprensión de su presente histórico que a su juicio no estaba a la altura de los postulados de la llustración. Por ello no sólo Feuerbach sino la izquierda hegeliana en general trata de conectar de nuevo con la tradición ilustrada, renaciendo así la figura de la Ilustración insatisfecha a la que había pretendido dar satisfacción Hegel. En concreto por lo que atañe a Feuerbach cabría decir que se encuentra en su momento de máxima aproximación a la Ilustración, aproximación que tendrá que hacer compatible con la herencia hegeliana.

48 Ibid., 176.

${ }^{49}$ G. W. VI, 17. 
Por lo demás, y en sintonía con la temática de esta monografía, también el estilo de Feuerbach cambia profundamente respecto a las aportaciones historiográficas anteriores. Se vuelve ahora mucho menos académico, mostrándose por el contrario mucho más libre e inconvencional. Feuerbach se siente liberado de las constricciones académicas, y lo mismo que Bayle busca un lenguaje más libre fomentando a su manera la causa de la filosoffa y de la "humanidad". La vigencia del legado ilustrado condiciona así el horizonte de los planteamientos historiográficos feuerbachianos.

\section{Feuerbach, Hegel y la filosofía alemana}

Cabría concluir esta aproximación a la concepción historiográfica de Feuerbach haciendo una referencia a su visión de la tradición filosófica alemana de la que él formaba parte y de la que en definitiva iba a constituir un nuevo capítulo, un capítulo sin duda que iba a tener un carácter epocal. El hecho de que Alemania estuviera a punto de concluir una de las etapas más brillantes de toda la historia de la filosofia. Cuando Feuerbach entra en acción, no podía menos de incidir en su conciencia historiográfica. Esta circunstancia le iba a conducir en definitiva a una valoración de la Modernidad filosófica alemana. No en vano los grandes representantes del Idealismo habían llamado la atención, de una u otra forma, sobre el papel especial desempeñado por Alemania en el desarrollo de la filosofía moderna. De un modo particular había insistido en ello su maestro Hegel para quien el pueblo alemán tendría algo así como la vocación superior de conservar el fuego sagrado de la filosofía en medio de la crisis generalizada de la misma en el seno de la cultura moderna ${ }^{50}$.

Sin duda la curiosidad feuerbachiana respecto a la Modernidad filosófica es muy amplia. Ante todo, siguiendo la tradición de Hegel y sus discípulos - y que en realidad ya provenía de la propia Ilustración - también muestra Feuerbach un gran interés por la tradición filosófica francesa y por toda su cultura en gene$\mathrm{ral}^{\mathrm{5}}{ }^{\mathrm{i}}$. Muestra asimismo un gran interés por la filosofía de la naturaleza del Renacimiento italiano, en especial por la de G. Bruno. Valoró altamente la filosofía de F. Bacon sobre todo en lo relativo al valor de la experiencia y de la ciencia. Por

\footnotetext{
${ }^{50}$ G. W. F. Hegel, Einleitung in die Geschichte der Philosophie, 4-5.

${ }^{51}$ Cf. A. Philonenko I, op. cit., 12.
} 
último resulta asimismo ineludible referirse a la fascinación que Feuerbach va a experimentar por la filosofía de Spinoza, siguiendo la tónica tan reiterada en la filosofía y en la literatura alemanas desde los tiempos de Lessing.

Todo ello no sólo ha despertado el interés de Feuerbach como historiador de la filosofía sino que ha incidido además en la constitución de su propia filosofía, de acuerdo con el talante general de la obra feuerbachiana. Pero de todas formas, Feuerbach va a prestar una especial atención a su propia tradición filosófica, la tradición alemana. Ya nos hemos referido a la confesión de Feuerbach según la que se consideraba heredero de Lutero y de Lessing, de Kant y de Fichte, de Goethe y de Hegel. Además no sólo se refiere con frecuencia a la tradición filosófica alemana en general sino que también lo hace a los grandes representantes de la literatura alemana.

La monografía dedicada al primer gran filósofo moderno alemán, Leibniz, le brinda a Feuerbach la oportunidad de reflexionar sobre algunas peculiaridades de la vía alemana a la filosofía. Alemania vendría a constituir el destino del complejo peregrinaje filosófico que para Feuerbach a estas alturas no habría duda de que habría comenzado propiamente en Italia. La filosofía moderna sería italiana de nacimiento pero sólo de nacimiento: «El italiano pudo sin duda engendrar la filosofía, pero el educarla, desarrollarla, determinarla y el formarla fue cometido de otros pueblos" ${ }^{52}$. Para Feuerbach no deja de tener un claro valor simbólico el hecho de que personajes como Bruno o Campanella se vieran precisados a buscar refugio en otros países europeos.

De una forma esquemática alude Feuerbach al peregrinaje de esa filosofía moderna por países como Inglaterra cuyo empirismo y utilitarismo se le antojaban demasiado estrechos, conservando la tradición metafísica sólo un valor histórico. A pesar de todo el acercamiento de Feuerbach al empirismo, su orientación filosófica propugna una mediación entre lo racional y lo empírico que le conduce más allá de las tesis puramente empirístas. Por ello centra su atención en la aportación francesa a la consolidación de la filosofía moderna. Los franceses se habrían enfrentado a la filosofía con un nuevo talante, más abierto a la dimensión metafísica, pero sólo a medias habría solucionado el problema, abo-

${ }^{52}$ G. W. III, 7. 
cando a una situación escindida entre posiciones idealistas y materialistas, lejos de una equilibrada mediación. Ante esta actitud desintegradora va a ser Spinoza, en Holanda, quien coja el testigo en el proceso evolutivo de la filosofía moderna. Spinoza como la libertas cogitandi personificada, logra a los ojos de Feuerbach esquivar los escollos extremistas a que se veía abocada la filosofía en Francia. $\mathrm{Va}$ a ser, no obstante, en Alemania donde se asiente la filosofía moderna, de una forma estable, después de su periplo por esos diversos países. Sería propiamente Alemania la que hereda las distintas aproximaciones a la nueva filosofía que se habían ido esbozando a lo largo de la Edad Moderna ${ }^{53}$. Alemania se habría sumado con retraso a la configuración de la filosofía moderna pero en compensación habría contribuido de una forma relevante a darle estabilidad y madurez.

Sin duda no ignora Feuerbach que Alemania ya había entrado tempranamente en contacto con la filosofía a través de la Reforma. En todos sus escritos historiográficos figura la referencia explícita al sentido y al influjo de la Reforma. Feuerbach es en realidad uno de los autores más representativos en el tema "protestantismo y filosofía", un autor que no dudará en autocalificarse como "Lutero II". De ahí que ya en el primer volumen de su Historia aparezca esbozado el doble principio moderno de la subjetividad, incoado por Lutero y Descartes. Tampoco en la monografía sobre Leibniz, al abordar el problema de la especificidad de la vía alemana hacia la filosofía, podía faltar dicha referencia, si bien ahora se alude abiertamente a la tensión inicial entre la doctrina luterana y la filosofía. Dicha tensión se irá, no obstante, mitigando y la nueva orientación religiosa va a constituir el horizonte en el que se va a configurar la filosofía alemana.

Ello va a marcar el destino de la filosofía alemana. Mientras en otros países como Francia, Inglaterra o Italia la filosofía va a afirmar su autonomía al margen de la religión establecida, a la que deja incólume, produciéndose una especie de escisión entre un universo filosófico donde la fe queda excluida y un mundo de la fe donde la razón no estaría autorizada a intervenir, en Alemania por el contrario se postularía una mediación consciente entre la filosofía y la religión existente, produciéndose una peculiar simbiosis entre ambos polos. He aquí una circunstancia que va a marcar el destino de la filosofía alemana. De este modo

53 Ibid., 9-10. 
filosofías como las de Leibniz o de Hegel resultarían incomprensibles sin tener presente esa mediación. Y si bien este hecho se va a convertir en uno de los flancos por los que Feuerbach va a cuestionar la tradición filosófica alemana, por otra parte la propia filosofía feuerbachiana no resultaría comprensible al margen de esta circunstancia.

Asimismo el estudio de Leibniz le hace reparar a Feuerbach en un segundo proceso mediador que también mostraría la especificidad de la filosofía alemana. Se trata de la conciliación de la modernidad y la tradición, de lo viejo y lo nuevo. Mientras en otros países la afirmación de la filosofía moderna implicaba una quiebra de la tradición, un rechazo y cuestionamiento de la filosof́a antigua, tal como ejemplifican autores como Descartes y Bacon, Leibniz, por el contrario, como primer gran representante de la filosofía alemana moderna, se va a esforzar por conciliar la filosofía antigua y la moderna. En vez de rechazar sin más sería preciso mejorar y prolongar la línea iniciada en el pasado ${ }^{54}$. Esta actitud supone para Feuerbach una mayor madurez por parte de la filosofía moderna, que ya estaría en condiciones de hacer justicia al pasado. Tal actitud llegaría a su consumación en el caso de Hegel que lleva a su plenitud el desarrollo de la filosofía moderna y a la vez establece un diálogo profundo con toda la tradición filosófica, comenzando por los griegos.

Es precisamente esta capacidad hegeliana para dialogar con la tradición un rasgo que va a impresionar profundamente a Feuerbach, tal como aparecía ya en la recensión de la Historia de la filosoffa de Hegel. Muchos son sin duda los grandes autores alemanes de los que Feuerbach se siente feudatario, tal como él mismo se encargó de recordar. Aparte de Lutero cabe afirmar que ocupan un lugar destacado Lessing, la figura prócer de la Ilustración alemana, a la que Feuerbach se va a referir repetidas veces en un sentido aprobatorio, y cuya Educación del género humano va a seguir proyectando su sombra sobre el proyecto feuerbachiano. Feuerbach fue bien consciente de que su obra se situaba no sólo en la prolongación de la obra luterana sino también de la inflexión llevada a cabo por Lessing. Algo similar cabría afirmar del primer gran representante del Idealismo alemán, Kant, cuya revolución tanto en el orden de la teoría del conocimiento como de la moral tan presente está en la obra feuerbachiana, por muchos que

${ }^{54}$ G. W. III, 15-16. 
sean los reparos que Feuerbach ponga a la vez ${ }^{55}$. Sea suficiente aquí con resaltar por último que el principal maestro de Feuerbach, Hegel, era quien ofrecía una especie de consumación de toda la tradición filosófica alemana, en la que por cierto iban a ocupar un lugar relevante las tres figuras que acabamos de destacar: Lutero, Lessing y Kant.

Ya en su madurez, Feuerbach seguía reconociendo: "Yo tuve una relación más íntima e influyente con Hegel que con cualquiera de nuestros antecesores espirituales" ${ }^{56}$, si bien quiere matizar en seguida que Hegel no fue para él una especie de Jehová o Alá filosóficos sino más bien el Zeus de la filosofía moderna que le dejaba la libertad suficiente para entusiasmarse por otros grandes representantes de la filosofía moderna.

En efecto, Feuerbach iba a dar pronto muestras de su independencia filosófica, por muy considerable que fuera el peso de las enseñanzas del maestro. Asimismo muy pronto iba a expresar la duda de si con Hegel no se habría consumado una época y se estaría abriendo paso otra nueva: “¿Cómo se relaciona la filosofía hegeliana con el presente y el futuro? ¿No es ella el mundo pasado como mundo intelectual? ¿Es ella algo más que un recuerdo de la humanidad de lo que ésta fue pero ya no es más?" $"$ "

Es cierto, a pesar de todo, que la incidencia de la filosofía hegeliana en el pensamiento de Feuerbach va a seguir siendo más fuerte de lo que permitirían pensar las tempranas dudas y cuestionamientos ${ }^{58}$. La propia recensión de la Historia de la filosofía así parece confirmarlo, por más que no podamos olvidar que fue publicado en el órgano de la Escuela hegeliana ni tampoco las expectativas profesionales que entonces abrigaba Feuerbach y que tenían una incidencia en la orientación de sus trabajos. Ciñiéndonos aquí a los escritos historiográficos, podemos observar tanto la creciente autonomía frente a Hegel como también su

55 A este respecto véanse entre otros los trabajos de P. Cerezo, "Un paralelo entre Kant y Feuerbach", en Revista de Filosofia, C .S .I .C ., enero-junio 1979, 5-32; M. Cabada, Feuerbach y Kant: dos actitudes antropológicas, Universidad Pontificia de Comillas, Madrid 1980.

56 L. Feuerbach, "Verhältnis zu Hegel", 509.

57 G. W. X, 156.

${ }^{58} \mathrm{Cf}$. W. Jaeschke «Feuerbach redivivus. Eine Auseinandersetzung mit der gegenwärtigen Forschung", en Hegel-Studien 13(1978), 202. 
rechazo de las descalificaciones demasiado acríticas de que era objeto la filosofía hegeliana en los años siguientes a la muerte del maestro. Sirva como ejemplo lo manifestado en la monografia sobre Leibniz. Por un lado Feuerbach señala que su exposición es independiente de lo que Hegel y otros habían dicho en favor o en contra de Leibniz. Pero cuando en algún pasaje discrepa abiertamente de lo afirmado por Hegel, se cuida de matizar que no se opone a él por seguir la "moda" $"$.

$\mathrm{Va}$ a ser precisamente la existencia de críticas poco rigurosas a la filosofía de Hegel, junto con la veneración sentida hacia su viejo maestro, lo que va a impedir que Feuerbach manifieste más abiertamente su distanciamiento crítico frente a los planteamientos hegelianos. Pero finalmente esa crítica va a emerger en el escrito Zur Kritik der Hegelschen Philosophie de 1839, que marca un hito en la evolución de la izquierda hegeliana. La crítica feuerbachiana va a afectar también a la concepción historiográfica hegeliana. A la muerte de Hegel se ha iniciado un nuevo periodo histórico y ello va a afectar a la vez a las concepciones historiográficas.

Sin duda Feuerbach se sigue sintiendo fascinado por la filosofía de Hegel. La historia de los sistemas filosóficos vendría a ser algo así como la pinacoteca de la razón en la que los grandes pensadores van dejando constancia de su percepción de la verdad. Pues bien Hegel vendría a ocupar un lugar especial en esta pinacoteca: «Hegel es el artista filosófico consumado, sus exposiciones constituyen muestras insuperables del sentido artístico cientificon ${ }^{60}$. Sin duda esta valoración se hacía extensiva a la condición de Hegel como magistral historiador de la filosofía.

Pero Feuerbach, que experimentó un influjo tan profundo de Hegel en lo concerniente al problema de la historicidad de la filosofía, va a denunciar ahora las insuficiencias del modelo historiográfico hegeliano. Ello va a tener lugar dentro del marco de la concepción hegeliana del tiempo. Feuerbach le va a criticar a Hegel que "sobredimensione» la relevancia del tiempo e infravalore por el contrario la del espacio. Ello le va a conducir a una sobrevaloración de la subordinación y de la sucesión en la consideración de las distintas formas de existencia.

\footnotetext{
59 G. W. III, 214-15.

${ }^{60}$ G. W. X, 32.
} 
Tal planteamiento conduciría a Hegel a una especie de teleologismo extremo de acuerdo con el que las formas inferiores pasarían necesariamente a las superiores. En este sentido Feuerbach vendría a coincidir con el diagnóstico de N. Hartmann cuando contrasta la teleología aristotélica que se esfuerza por hacer justicia a los fenómenos con la hegeliana que se movería en el horizonte de una racionalismo absoluto ${ }^{61}$. Por supuesto tal valoración sería aplicable asimismo a la consideración hegeliana de los sistemas filosóficos reducidos a la condición de "momentos» de la filosofía posterior. También a este respecto cabría hablar de un teleologismo extremo, con el sometimiento excesivo de unas manifestaciones filosóficas a otras a través de la historia.

Por otra parte Feuerbach también se hace eco de la sensación que tuvo la época, propiciada en algún sentido por el propio Hegel, de que en la filosofia hegeliana asistiríamos a algo así como a la consumación de la filosofía, al presentarse como la "filosofía misma", como la filosofía absoluta. Se trataría de una especie de plenitud escatológica que de alguna manera implicaría el "fin" de la historia. Evidentemente tanto en este aspecto como en el anterior serían necesarias más precisiones y matizaciones de las que ofrece Feuerbach para no simplificar indebidamente el pensamiento de Hegel, pero en todo caso la crítica feuerbachiana acierta a la hora de señalar algunos de los aspectos más cuestionables de la concepción hegeliana. Tal es el caso cuando por un lado denuncia la presencia excesiva de una lógica de la subordinación de unas formas a otras, y por otro cuando critica la tendencia a la absolutización de una manifestación filosófica como es la filosofía hegeliana. Es decir, por una parte se atribuirfa un valor excesivo al tiempo, a la temporalización de la existencia y por otra asistiríamos a la neutralización del tiempo en cuanto consumación del mismo. De esta forma, una vez que Feuerbach critica la abusiva teleologia hegeliana, reivindica frente al maestro la apertura del tiempo y de la historia, en un momento en que se estaba iniciando un nuevo periodo histórico. Feuerbach acaba temporalizando al gran maestro de la temporalidad que tanto había influido en él. La filosofia de Hegel, como todo lo que entra en el espacio y en el tiempo, debe someterse a las leyes que supone este hecho: «El deus terminus actúa como vigilante a la entrada del mundo" ${ }^{62}$. También la filosofía hegeliana habría tenido que pagar este tributo.

${ }^{61}$ N. Hartmann, «Aristoteles und Hegel, en Kleinere Schriften II, W. de Gruyter, Berlin 1957, 214-253.

${ }^{62}$ G. W. X, 20. 
Al mismo tiempo se reivindicaba el derecho a la existencia de la filosofía después de Hegel.

Precisamente en esta reivindicación va a insistir Feuerbach en otro escrito del mismo año 1839 titulado Über Philosophie und Christentum. En dicho escrito Feuerbach va a polemizar con el historiador $\mathrm{H}$. Leo que había atacado el rumbo emprendido por la izquierda hegeliana. Feuerbach cuestiona una concepción de la historia que se cierra al cambio y a la innovación. Tal sería el caso del historiador Leo que expresa su reconocimiento por el viejo Hegel que sería para él un verdadero filósofo porque "ha cesado de filosofar», y también serían verdaderos filósofos los discípulos ortodoxos del maestro que se limitan a reiterar lo que él ya ha dicho, a conservar fielmente su herencia espiritual. No lo serían por el contrario aquellos otros discípulos de Hegel que no renuncian a pensar por sí mismos más allá de lo pensado por el maestro ${ }^{63}$. Feuerbach se aprestaba a liderar esa nueva etapa de la filosofía alemana, que va a constituir el comienzo de la filosofía contemporánea como tal. Los próximos ańos en los que va a publicar Das Wesen des Christentums y toda una serie de manifiestos acerca de una nueva filosofía así lo vienen a confirmar. Pero este avanzar más allá de Hegel iba a ir acompañado del movimiento de conectar con una serie de movimientos y tendencias prehegelianos que Hegel habia intentado subsumir de una forma demasiado expeditiva en su sistema. Asi ocurría con el legado de la Ilustración y la orientación antropológica de la filosofia kantiana. De esta forma el proyecto de una filosofía del futuro no se hace al margen de la revisión de la visión historiográfica hegeliana, y de una nueva aproximación a la tradición filosófica.

${ }^{63}$ G. W. VIII, 229. 